\title{
A simple modification to lower incidence of heart block with sutureless valve implantation
}

\author{
Bobby Yanagawa, MD, PhD, Jorge Cruz, MD, Lyna Boisvert, RN, CV-ICU, BScN, and \\ Daniel Bonneau, MD, Toronto, Ontario, Canada
}

\footnotetext{
From the Division of Cardiac Surgery, St Michael's Hospital, University of Toronto, Toronto, Ontario, Canada. B.Y. and J.C. contributed equally.

Disclosures: Authors have nothing to disclose with regard to commercial support.

Received for publication Nov 30, 2015; revisions received Feb 5, 2016; accepted for publication Feb 16, 2016; available ahead of print March 10, 2016.

Address for reprints: Daniel Bonneau, MD, Division of Cardiac Surgery, St Michael's Hospital, 30 Bond St, 8th

Floor, Bond Wing, Toronto, Ontario, M5B 1W8 Canada (E-mail: bonneaud@smh.ca).

J Thorac Cardiovasc Surg 2016;152:630-2

$0022-5223 / \$ 36.00$

Copyright (c) 2016 by The American Association for Thoracic Surgery

http://dx.doi.org/10.1016/j.jtcvs.2016.02.034
}

The Perceval (LivaNova, Milan, Italy) is a sutureless bovine pericardial valve mounted on a Nitinol stent (Figure 1, top). ${ }^{1}$ According to the manufacturer's instructions, 3 guiding sutures should be placed 2 to $3 \mathrm{~mm}$ beneath the leaflet insertion line, which are threaded into the eyelets of the valve to stabilize position for deployment. A major drawback to the Perceval valve is a relatively high rate of conduction disturbance necessitating permanent pacemaker (PPM) implantation. ${ }^{2-5}$ In our initial experience (cohort 1), we report a 28\% (7/25 patients) rate of pacemaker implantation. This was reduced to $0 \%(0 / 18$ patients; cohort 2) with a simple technical modification of placing the guiding sutures at the leaflet insertion line.

\section{CLINICAL SUMMARY}

Between November 2013 and November 2015, 43 patients underwent aortic valve replacement (AVR) with the Perceval valve; $67 \%$ received combined AVR + coronary artery bypass grafting, and 33\% received isolated AVR. Approval for this project was obtained from the St Michael's Hospital Research Ethics Board. Individual patient consent was waived. Cohort $1(n=25)$ represents patients before the modification, and cohort $2(\mathrm{n}=18)$ represents patients after the modification. The mean age was $75.3 \pm 8.6$ years, and the mean Society of Thoracic Surgeons Predicted Risk of Mortality was $3.7 \pm 2.3$. Sixteen percent of patients were diabetic, $5 \%$ were reoperative procedures, and $16 \%$ of patients had a bicuspid aortic valve. With regard to rhythm status, $63 \%$ of patients had sinus rhythm, $14 \%$ of patients had atrial fibrillation, $9 \%$ of patients had right bundle branch block, and $7 \%$ of patients had first-degree block. The mean time from aortotomy to closure of aortotomy (AVR duration) was $29.9 \pm 10.6$ minutes.

There were 4 operative mortalities: One patient with biventricular failure undergoing reoperative AVR was unable to be weaned from cardiopulmonary bypass; 1 patient died of ischemic gut and sepsis; 1 patient with Child-Pugh

\section{DISCUSSION}

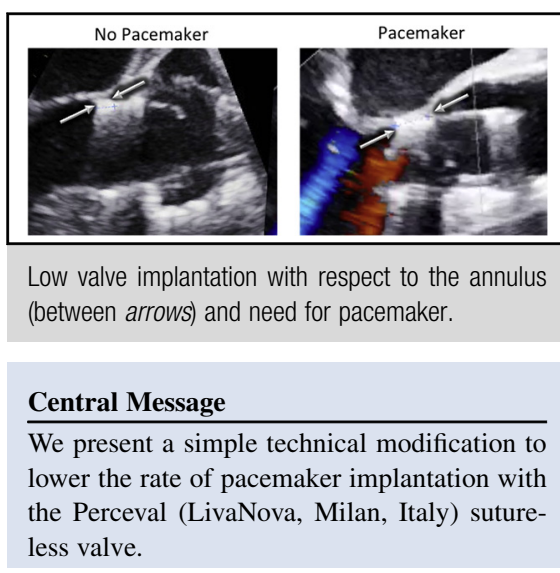

See Editorial Commentary page 633.

B cirrhosis died of liver failure; and 1 patient with previous colon resection died of ileus and bowel perforation.

In cohort 1, 14 patients required temporary pacing (56\%) and 7 patients had heart block requiring a PPM (28\%). At this point, we performed an internal review of our series. There was no association between PPM implantation and preoperative rhythm; 1 patient was in first-degree heart block, 1 patient had right bundle branch block, 1 patient was in atrial fibrillation, and 4 patients were in sinus rhythm. Echocardiographically, there was a significantly greater distance from the aortic annulus to the lower aspect of the sealing collar for patients with PPM than those without PPM $(7.3 \pm 1.5 \mathrm{~mm}$ vs $4.7 \pm 1.0 \mathrm{~mm}, P=.001)$ (Figure 1, middle and bottom). Therefore, we modified our technique by placing the guiding sutures at the nadir of each cusp and not 2 to $3 \mathrm{~mm}$ below. Postmodification (cohort 2), 6 patients required temporary pacing $(33.3 \%)$ and the PPM rate was $0 \%(0 / 18)$. There was 1 case of mild paravalvular leak in each of cohorts 1 and 2 . The mean time for PPM implantation was postoperative day $7.3 \pm 4.0$. There was no difference in in-hospital length of stay compared with patients who did not require PPM (mean: $8.7 \pm 2.0$ days vs $10.7 \pm 8.8$ days, $P=.6$; median 9.0 vs 7.5 days). At a mean follow-up of $198 \pm 177$ days, 5 of 7 patients with PPM were pacemaker dependent.

Several groups have reported high rates of pacemaker implantation with the Perceval valve compared with 

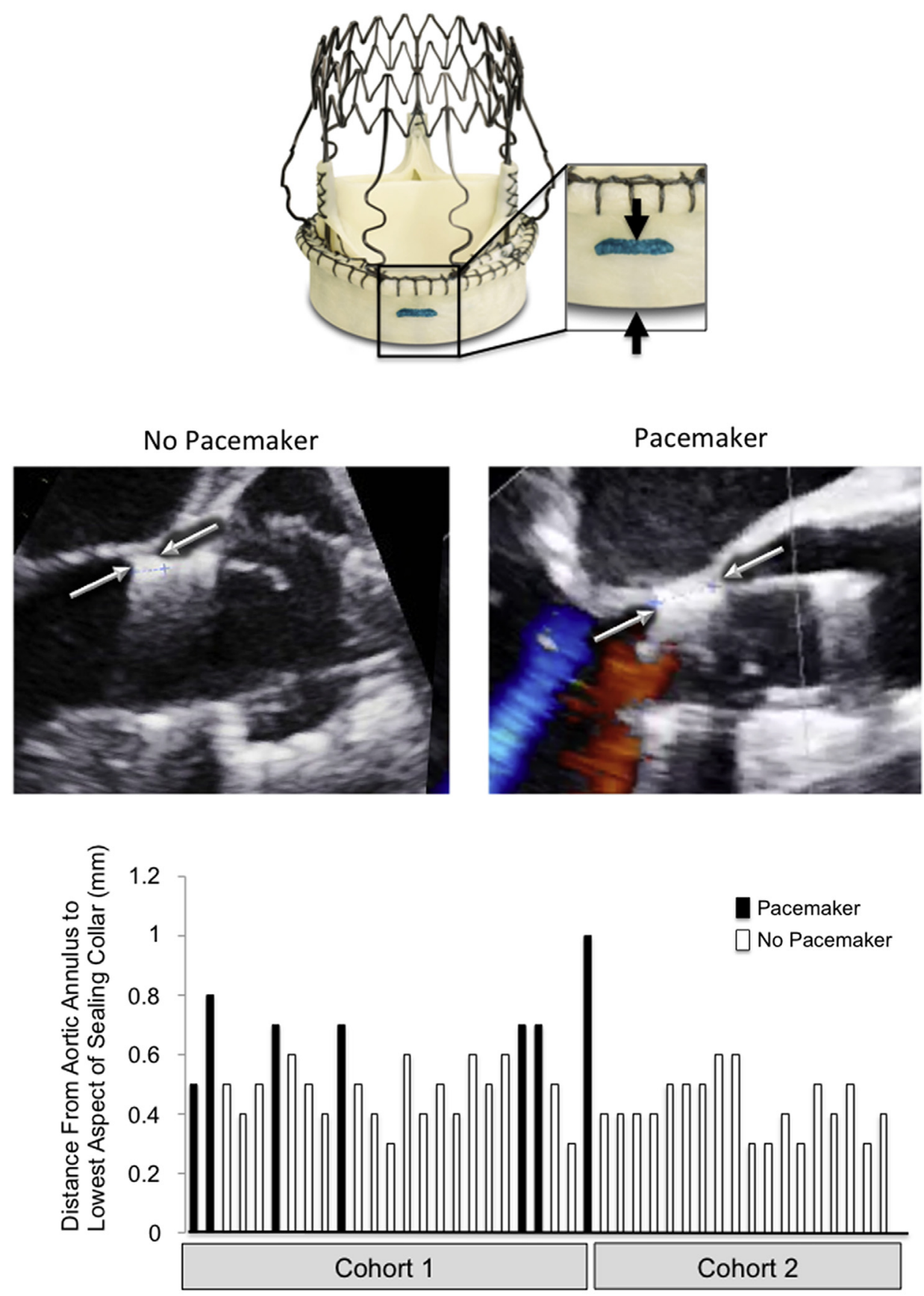

Patients

FIGURE 1. Top: Image of the Perceval sutureless valve (courtesy of LivaNova, Milan, Italy) and the width from the eyelet to the sealing collar edge (insert, between arrows). Middle: Transesophageal echocardiographic image showing the distance from the annulus to the lower edge of the sealing collar (between arrows): $4 \mathrm{~mm}$ in a patient without PPM (left) and $10 \mathrm{~mm}$ in a patient with PPM for heart block (right). Bottom: Distance from annulus to lower edge of sealing collar for all patients.

conventional AVR. ${ }^{2-5}$ The 5-year experience from 3 European multicenter trials $(\mathrm{N}=731)$ reported a $7.4 \%$ rate of PPM for heart block. ${ }^{2}$ The Canadian experience of 215 patients reported 17\% PPM implantation. ${ }^{3}$ In 658 patients in the European Multicentre Cavalier Trial, the incidence rate of PPM implantation was $11.6 \%$ overall and $6.7 \%$ in patients without preoperative cardiac rhythm disturbances. ${ }^{4}$

In our experience, the lower aspect of the sealing collar more than $6 \mathrm{~mm}$ below the annulus was associated with the need for PPM. According to the manufacturer, the distances between the eyelet (green) and bottom of the 
TABLE 1. Perceval (LivaNova, Milan, Italy) valve sizes in the overall population (overall) and in patients requiring temporary pacing and implantation of permanent pacemaker

\begin{tabular}{lccl}
\hline Size & Overall & Temporary pacing $(\%)$ & PPM $(\%)$ \\
\hline S & 10 & $4(40)$ & $2(20.0)$ \\
M & 13 & $5(38.4)$ & $2(15.4)$ \\
L & 13 & $6(46.2)$ & $1(7.7)$ \\
XL & 7 & $5(71.4)$ & $2(28.6)$ \\
\hline
\end{tabular}

$P P M$, Permanent pacemaker; $S$, small; $M$, medium; $L$, large; $X L$, extra large.

sealing collar are $3.9 \mathrm{~mm}$ (small), $4.2 \mathrm{~mm}$ (medium), $4.5 \mathrm{~mm}$ (large), and $4.8 \mathrm{~mm}$ (extra large) (Figure 1, top). In our series, the extra large size was associated with a $71.4 \%$ and $28.6 \%$ need for temporary pacing and PPM implantation, respectively, compared with $46.5 \%$ and $16 \%$ overall, respectively (Table 1 ). These numbers were too small to make any firm association between valve size and need for pacemaker. Given that lower implantation was associated with incidence of heart block, it is tempting to speculate that subannular expansion of the sealing collar and compression of conduction tissue, such as the bundle of His, may be the culprit. However, conduction disturbances with prosthetic valve implantation are multifactorial, and more work is needed to confirm these findings and to fully elucidate the mechanism. Of note, our modification was not associated with increased incidence of paravalvular leak. There were no changes in our protocol for valve sizing, balloon expansion, or calcium debridement between the initial and latter cohorts. Finally, the overall mortality in our study was higher than the Society of Thoracic Surgeons Predicted Risk of Mortality, largely due to nonvalve-related causes.

\section{CONCLUSIONS}

On the basis of these data, low valve positioning may contribute to conduction block and need for PPM with the Perceval sutureless valve. Insertion of the guiding suture at the nadir of the aortic annulus may help to avoid conduction disturbances. Our rate of PPM implantation postmodification compared favorably to published PPM rates for surgical AVR. ${ }^{5}$

\section{References}

1. Gersak B, Fischlein T, Folliguet TA, Meuris B, Teoh KH, Moten SC, et al. Sutureless, rapid deployment valves and stented bioprosthesis in aortic valve replacement: recommendations of an International Expert Consensus Panel. Eur J Cardiothorac Surg. 2016;49:709-18.

2. Shrestha M, Fischlein T, Meuris B, Flameng W, Carrel T, Madonna F, et al. European multicentre experience with the sutureless Perceval valve: clinical and haemodynamic outcomes up to 5 years in over 700 patients. Eur J Cardiothorac Surg. 2016;49:234-41.

3. Mazine A, Teoh K, Bouhout I, Bhatnagar G, Pelletier M, Voisine P. Sutureless aortic valve replacement: a Canadian multicentre study. Can J Cardiol. 2015; 31:63-8.

4. Laborde F, Fischlein T, Hakim-Meibodi K, Misfeld M, Carrel T, Zembala M, et al. Clinical and haemodynamic outcomes in 658 patients receiving the Perceval sutureless aortic valve: early results from a prospective European multicentre study (the Cavalier Trial). Eur J Cardiothorac Surg. 2016;49: 978-86.

5. Dawkins S, Hobson AR, Kalra PR, Tang AT, Monro JL, Dawkins KD. Permanent pacemaker implantation after isolated aortic valve replacement: incidence, indications, and predictors. Ann Thorac Surg. 2008;85:108-12. 